\title{
On the Cauchy Problem for a Simple Degenerate Diffusion System
}

\author{
By \\ Masayasu MimurA*
}

\section{§ 1. Introduction}

We consider the following Cauchy problem for the system of equations :

$$
\begin{aligned}
& \frac{\partial u_{1}}{\partial t}=\frac{\partial^{2} u_{1}}{\partial x^{2}}-d_{1} u_{1} u_{4}-d_{2} u_{1} u_{3} \\
& \frac{\partial u_{2}}{\partial t}=\frac{\partial^{2} u_{2}}{\partial x^{2}}-d_{3} u_{2} u_{4}+d_{2} u_{1} u_{3} \\
& \frac{\partial u_{3}}{\partial t}=\quad d_{3} u_{2} u_{4}-d_{2} u_{1} u_{3} \\
& \frac{\partial u_{4}}{\partial t}=\quad-d_{1} u_{1} u_{4}-d_{3} u_{2} u_{4}
\end{aligned}
$$

in $R^{T}=\left(-\infty<x<+\infty, 0<t \leqslant^{V} T\right)$ with the initial data

$$
\begin{aligned}
& u_{1}(x, 0)=u_{10}(x) \\
& u_{2}(x, 0)=u_{20}(x) \\
& u_{3}(x, 0)=u_{30}(x) \\
& u_{4}(x, 0)=u_{40}(x)
\end{aligned}
$$

where the initial data are all non-negative and the coefficients $d_{1}, d_{2}$ and $d_{3}$ are positive constants.

Such a system is considered one of the problems of diffusion accompanied by an immobilizing reaction of second order (2), (4).

Received March 13, 1969.

Communicated by S. Matsuura.

* Department of Applied Mathematics and Physics, Faculty of Engineering, Kyoto University. 
In this paper, we intend to prove the uniqueness and the existence of the solution of the problem (1.1) and (1.2) by using a suitable simple difference scheme.

Using the following vector and matrix notations,

$$
\begin{aligned}
U & ={ }^{t}\left(u_{1}, u_{2}, u_{3}, u_{4}\right), \\
D & =\left(\begin{array}{llll}
1 & 0 & 0 & 0 \\
0 & 1 & 0 & 0 \\
0 & 0 & 0 & 0 \\
0 & 0 & 0 & 0
\end{array}\right), \\
A(U) & =\left(\begin{array}{rrrr}
-d_{2} u_{3} & 0 & 0 & -d_{1} u_{1} \\
d_{2} u_{3} & -d_{3} u_{4} & 0 & 0 \\
-d_{2} u_{3} & d_{3} u_{4} & 0 & 0 \\
0 & -d_{3} u_{4} & 0 & -d_{1} u_{1}
\end{array}\right),
\end{aligned}
$$

the system (1.1) and (1.2) is reduced to

$$
\begin{aligned}
& \frac{\partial U}{\partial t}=D \frac{\partial^{2} U}{\partial x^{2}}+A(U) U, \\
& U(x, 0)=U_{0}(x) .
\end{aligned}
$$

We introduce the following difference scheme to (1.1) and (1.2),

$$
\begin{aligned}
& u_{1}^{n+1, j}=P\left(u_{1}^{n, j}\right)-k\left(d_{1} u_{1}^{n+1, j} u_{4}^{n, j}+d_{2} u_{1}^{n+1, j} u_{3}^{n, j}\right) \\
& u_{2}^{n+1, j}=P\left(u_{2}^{n, j}\right)-k\left(d_{3} u_{2}^{n+1, j} u_{4}^{n, j}-d_{2} u_{1}^{n+1, j} u_{3}^{n, j}\right) \\
& u_{3}^{n+1, j}=u_{3}^{n, j}+k\left(d_{3} u_{2}^{n, j} u_{4}^{n+1, j}-d_{2} u_{1}^{n, j} u_{3}^{n+1, j}\right) \\
& u_{4}^{n+1, j}=u_{4}^{n, j}-k\left(d_{1} u_{1}^{n, j} u_{4}^{n+1, j}+d_{3} u_{2}^{n, j} u_{4}^{n+1, j}\right)
\end{aligned}
$$

in $R_{h}^{T}=$ (the rectangular lattices with mesh sizes $(h, k)$ in $R^{T}$ ) with the initial data

$$
\begin{aligned}
& u_{1}^{0, j}=u_{10}(j h) \\
& u_{2}^{0, j}=u_{20}(j h) \quad \text { or } \quad U^{0, j}=U_{0}(j h), \\
& u_{3}^{0, j}=u_{30}(j h) \\
& u_{4}^{0, j}=u_{40}(j h)
\end{aligned}
$$

where $U^{n, j}=U(j h, n k)$ for integers $j$ and $n(0 \leqslant n k \leqslant T), P\left(u_{i}^{n, j}\right)$ $=\lambda u_{i}^{n, j-1}+(1-2 \lambda) u_{i}^{n, j}+\lambda u_{i}^{n, j+1}$ for $i=1,2$ and $\lambda=k / h^{2}=$ const. 


\section{§. Existence Theorem}

The methods used in this section are essentially the same as those used by Fritz John [6].

First we have the following lemma.

Lemma 1 (Stability).

A sufficient condition for stability of the difference scheme (1.5) and (1.6) is given by the following inequality

$$
0<\lambda \leqslant \frac{1}{2} \text {. }
$$

Proof. We can write (1.5) as follows :

$$
\begin{aligned}
u_{1}^{n+1, j} & =\frac{P\left(u_{1}^{n, j}\right)}{1+k\left(d_{1} u_{4}^{n, j}+d_{2} u_{3}^{n, j}\right)} \\
u_{2}^{n+1, j} & =\frac{P\left(u_{2}^{n, j}\right)+k d_{2} u_{1}^{n+1, j} u_{3}^{n, j}}{1+k d_{3} u_{4}^{n, j}} \\
u_{3}^{n+1, j} & =\frac{u_{3}^{n, j}+k d_{3} u_{2}^{n, j} u_{4}^{n+1, j}}{1+k d_{2} u_{1}^{n, j}} \\
u_{4}^{n+1, j} & =\frac{u_{4}^{n, j}}{1+k\left(d_{1} u_{1}^{n, j}+d_{3} u_{2}^{n, j}\right)} .
\end{aligned}
$$

If $U^{0, j}$ is non-negative, it follows from (2.2) that $U^{n, j}$ is nonnegative for any $n$. Therefore we have from (1.5) that

$$
\begin{aligned}
& u_{1}^{n+1, j} \leqslant P\left(u_{1}^{n, j}\right), \\
& u_{1}^{n+1, j}+u_{2}^{n+1, j} \leqslant P\left(u_{1}^{n, j}+u_{2}^{n, j}\right), \\
& u_{3}^{n+1, j}+u_{4}^{n+1, j} \leqslant u_{3}^{n, j}+u_{4}^{n, j}, \\
& u_{4}^{n+1, j} \leqslant u_{4}^{n, j} .
\end{aligned}
$$

Thus we have the following estimates for any $n$,

$$
\begin{aligned}
& \left|u_{1}^{n}\right| \leqslant\left|u_{1}^{n}\right|, \\
& \left|u_{2}^{n}\right| \leqslant\left|u_{1}^{n}\right|+\left|u_{2}^{n}\right|, \\
& \left|u_{3}^{n}\right| \leqslant\left|u_{3}^{n}\right|+\left|u_{4}^{n}\right|, \\
& \left|u_{4}^{n}\right| \leqslant\left|u_{4}^{0}\right|,
\end{aligned}
$$

where

$$
\left|u_{i}^{n}\right|=\sup _{j}\left|u_{i}^{n, j}\right| \quad \text { for } \quad i=1,2,3,4 .
$$

Lemma 1 is proved. 
Secondly we can show that the $x$-difference quotient $U_{x}^{n, j}$ $=\left(U^{n, j+1}-U^{n, j}\right) / h$ satisfying (1.5) and (1.6) is bounded uniformly in $h$. In fact we have the following equations from (1.5) and (1.6),

$$
\begin{aligned}
\left(u_{1}^{n+1, j}\right)_{x}= & P\left(\left(u_{1}^{n, j}\right)_{x}\right)-k\left[d _ { 1 } \left\{u_{1}^{n, j+1}\left(u_{1}^{n+1, j}\right)_{x}\right.\right. \\
& \left.+u_{1}^{n+1, j}\left(u_{4}^{n, j}\right)_{x}\right\}+d_{2}\left\{u_{3}^{n, j+1}\left(u_{1}^{n+1, j}\right)_{x}\right. \\
& \left.\left.+u_{1}^{n+1, j}\left(u_{3}^{n, j}\right)_{x}\right\}\right], \\
\left(u_{2}^{n+1, j}\right)_{x}= & P\left(\left(u_{2}^{n, j}\right)_{x}\right)-k\left[d _ { 3 } \left\{u_{4}^{n, j+1}\left(u_{2}^{n+1, j}\right)_{x}\right.\right. \\
& \left.+u_{2}^{n+1, j}\left(u_{4}^{n, j}\right)_{x}\right\}-d_{2}\left\{u_{3}^{n, j+1}\left(u_{1}^{n+1, j}\right)_{x}\right. \\
& \left.\left.+u_{1}^{n+1, j}\left(u_{3}^{n, j}\right)_{x}\right\}\right], \\
\left(u_{3}^{n+1, j}\right)_{x}= & \left(u_{3}^{n, j}\right)_{x}+k\left[d _ { 3 } \left\{u_{2}^{n, j+1}\left(u_{4}^{n+1, j}\right)_{x}\right.\right. \\
& \left.+u_{4}^{n+1, j}\left(u_{2}^{n, j}\right)_{x}\right\}-d_{2}\left\{u_{1}^{n, j+1}\left(u_{3}^{n+1, j}\right)_{x}\right. \\
& \left.\left.+u_{3}^{n+1, j}\left(u_{1}^{n, j}\right)_{x}\right\}\right], \\
\left(u_{4}^{n+1, j}\right)_{x}= & \left(u_{4}^{n, j}\right)_{x}-k\left[d _ { 1 } \left\{u_{1}^{n, j+1}\left(u_{4}^{n+1, j}\right)_{x}\right.\right. \\
& \left.+u_{4}^{n+1, j}\left(u_{1}^{n, j}\right)_{x}\right\}+d_{3}\left\{u_{2}^{n, j+1}\left(u_{4}^{n+1, j}\right)_{x}\right. \\
& \left.\left.+u_{4}^{n+1, j}\left(u_{2}^{n, j}\right)_{x}\right\}\right],
\end{aligned}
$$

and also the initial conditions

$$
\left.U_{x}^{0, j}=U_{0}((j+1) h)-U_{0}(j h)\right) / h .
$$

Then it follows from (2.5) and Lemma 1 that

$$
\begin{aligned}
& \left|\left(u_{1}^{n+1}\right)_{x}\right| \leqslant !\left(u_{1}^{n}\right)_{x} !+k D M\left(\left|\left(u_{4}^{n}\right)_{x}\right|+\left|\left(u_{3}^{n}\right)_{x}\right|\right), \\
& \left|\left(u_{2}^{n+1}\right)_{x}\right| \leqslant\left|\left(u_{2}^{n}\right)_{x}\right|+k D M\left(\left|\left(u_{4}^{n}\right)_{x}\right|+\left|\left(u_{3}^{n}\right)_{x}\right|+\left|\left(u_{1}^{n+1}\right)_{x}\right|\right), \\
& \left|\left(u_{3}^{n+1}\right)_{x}\right| \leqslant\left|\left(u_{3}^{n}\right)_{x}\right|+k D M\left(\left|\left(u_{4}^{n+1}\right)_{x}\right|+\left|\left(u_{2}^{n}\right)_{x}\right|+\left|\left(u_{1}^{n}\right)_{x}\right|\right), \\
& \left|\left(u_{4}^{n+1}\right)_{x}\right| \leqslant\left|\left(u_{4}^{n}\right)_{x}\right|+k D M\left(\left|\left(u_{1}^{n}\right)_{x}\right|+\left|\left(u_{2}^{n}\right)_{x}\right|\right),
\end{aligned}
$$
where $M=\max _{i}\left\{\sup _{R_{h}^{T}}\left|u_{i}^{n, j}\right|\right\}$ and $D=\max \left(d_{1}, d_{2}, d_{3}\right)$. Therefore, we
obtain

where

$$
\begin{aligned}
& \left|U_{x}^{n}\right| \leqslant e^{3 D M T}\left|U_{x}^{0}\right|, \\
& \left|U_{x}^{n}\right|=\sum_{i=1}^{4}\left|\left(u_{i}^{n}\right)_{x}\right| .
\end{aligned}
$$

Consequently, if $U^{0, j}$ is of class $\mathscr{B}^{1}, U_{x}^{n, j}$ is bounded uniformly in $h$. Similarly, we have the following equations for $U_{t}^{n, j}$; 


$$
\begin{aligned}
\left(u_{1}^{n+1, j}\right)_{t}= & P\left(\left(u_{1}^{n, j}\right)_{t}\right)-k\left[d _ { 1 } \left\{u_{4}^{n+1, j}\left(u_{1}^{n+1, j}\right)_{t}\right.\right. \\
& \left.+u_{1}^{n+1, j}\left(u_{4}^{n, j}\right)_{t}\right\}+d_{2}\left\{u_{3}^{n+1, j}\left(u_{1}^{n+1, j}\right)_{t}\right. \\
& \left.\left.+u_{1}^{n+1, j}\left(u_{3}^{n, j}\right)_{t}\right\}\right], \\
\left(u_{2}^{n+1, j}\right)_{t}= & P\left(\left(u_{2}^{n, j}\right)_{t}\right)-k\left[d _ { 3 } \left\{u_{4}^{n+1, j}\left(u_{2}^{n+1, j}\right)_{t}\right.\right. \\
& \left.+u_{2}^{n+1, j}\left(u_{4}^{n, j}\right)_{t}\right\}-d_{2}\left\{u_{3}^{n+1, j}\left(u_{1}^{n+1, j}\right)_{t}\right. \\
& \left.\left.+u_{1}^{n+1, j}\left(u_{3}^{n, j}\right)_{t}\right\}\right], \\
\left(u_{3}^{n+1, j}\right)_{t}= & \left(u_{3}^{n, j}\right)_{t}+k\left[d _ { 3 } \left\{u_{2}^{n+1, j}\left(u_{4}^{n+1, j}\right)_{t}\right.\right. \\
& \left.+u_{4}^{n+1, j}\left(u_{2}^{n, j}\right)_{t}\right\}-d_{2}\left\{u_{1}^{n+1, j}\left(u_{2}^{n+1, j}\right)_{t}\right. \\
& \left.\left.+u_{3}^{n+1, j}\left(u_{1}^{n, j}\right)_{t}\right\}\right], \\
\left(u_{4}^{n+1, j}\right)_{t}= & \left(u_{4}^{n, j}\right)_{t}-k\left[d _ { 1 } \left\{u_{1}^{n+1, j}\left(u_{4}^{n+1, j}\right)_{t}\right.\right. \\
& \left.+u_{4}^{n+1, j}\left(u_{1}^{n, j}\right)_{t}\right\}+d_{3}\left\{u_{2}^{n+1, j}\left(u_{4}^{n+1, j}\right)_{t}\right. \\
& \left.\left.+u_{4}^{n+1, j}\left(u_{2}^{n, j}\right)_{t}\right\}\right],
\end{aligned}
$$

and the initial conditions

$$
U_{t}^{o, j}=\left(U^{1, j}-U_{0}(j h)\right) / k,
$$

where $U_{t}^{n, j}=\left(U^{n+1, j}-U^{n, j}\right) / k$. Hence we have the following estimate;

$$
\left|U_{t}^{n}\right| \leqslant e^{3 D M T}\left(\left|\left(u_{10}\right)_{x x}\right|+\left|\left(u_{20}\right)_{x x}\right|+8 D M^{2}\right) .
$$

Therefore, if $u_{10}, u_{20}, u_{30}$ and $u_{40}$ are of class $\mathscr{B}^{2}, \mathscr{B}^{2}, \mathscr{B}^{1}$ and $\mathscr{B}^{1}$ respectively, $U^{n, j}, U_{x}^{n, j}$ and $U_{t}^{n, j}$ are bounded uniformly in $h$ from (2.8) and (2.11). Proceeding in this manner we see that, if $u_{10}, u_{20}, u_{30}$ and $u_{40}$ are of class $\mathscr{B}^{4}, \mathscr{B}^{4}, \mathscr{B}^{3}$ and $\mathscr{B}^{3}$ respectively, all quantities

$$
U^{n, j}, U_{x}^{n, j}, U_{x x}^{n, j}, U_{x x x}^{n, j}, U_{t}^{n, j}, U_{x t}^{n, j}, U_{x x t}^{n, j}, U_{t i}^{n, j}
$$

are bounded uniformly in $h$.

Now we consider a sequence of lattices $R_{h_{m}}^{T}$ with mesh sizes $\left(h_{m}=h_{0} / 2^{m}, k_{m}=\lambda h_{m}^{2}\right)$ for a positive fixed number $h_{0}$ and non-negative integers $m$. Let define $R_{h_{\infty}}^{T}$ be the sum of all the sets $R_{h_{m}}^{T}$, then $R_{h_{\infty}}^{T}$ is a denumerable set and everywhere dense in $R^{T}$.

Since $U^{n, j}$ is bounded uniformly in $h$, it follows that there exists a sequence $I$ of non-negative integers $m$ such that

$$
\lim _{\substack{m \subset I \\ m \rightarrow \infty}} U_{m}^{n, j}=U(x, t) \quad \text { in } R_{h_{\infty}}^{T} .
$$

Here $U_{m}^{n, j}=U\left(j h_{m}, h k_{m}\right)$ is defined in $R_{h_{m}}^{T}$. If $U_{m}^{n, j}$ and $U_{m}^{n^{\prime}, j^{\prime}}$ are both 
defined in $R_{h_{m}}^{T}$ for large $m$, then from the boundness of $U_{x}^{n, j}$ and $U_{t}^{n, j}$,

$$
\begin{aligned}
U_{m}^{n^{\prime}, j^{\prime}}-U_{m}^{n, j} & =h_{m} \sum_{\alpha=1}^{\left|j^{\prime}-j^{\prime}\right|}\left(U_{m}^{n^{\prime}, j+\alpha-1}\right)_{x}+k_{m} \sum_{\beta=1}^{\left|n^{\prime}-n\right|}\left(U_{m}^{n+\beta-1, j}\right)_{t} \\
& =0\left(x-x^{\prime}\right)+0\left(t-t^{\prime}\right) \quad \text { in } R_{h_{m}}^{T},
\end{aligned}
$$

where $(x, t)=\left(j h_{m}, n k_{m}\right)$ and $\left(x^{\prime}, t^{\prime}\right)=\left(j^{\prime} h_{m}, n^{\prime} k_{m}\right)$. For $m \subset I$ and $m \rightarrow \infty$, we have

$$
U\left(x^{\prime}, t^{\prime}\right)-U(x, t)=0\left(x^{\prime}-x\right)+0\left(t^{\prime}-t\right) \quad \text { in } \quad R_{h_{\infty}}^{T} .
$$

Consequently the limit function $U(x, t)$ is of class $\mathcal{E}_{t}^{0}\left(\mathscr{G}^{0}\right)$ in $R_{h_{\infty}}^{T}$ and we can continue $U(x, t)$ into the whole set $R^{T}$. Then $U(x, t)$ is of class $\mathcal{E}_{t}^{0}\left(\mathscr{B}^{0}\right)$ in $R^{T}$.

Since all quantities of (2.12) are uniformly bounded, we conclude in the same discussion that $\left(U^{n, j}\right)_{x},\left(U^{n, j}\right)_{x x}$ and $\left(U^{n, j}\right)_{t}$ converge respectively towards the corresponding derivatives of $U(x, t)$ and that the limits are of class $\mathcal{E}_{t}^{0}\left(\mathscr{B}^{0}\right)$ in $R^{T}$.

Finally in the limit as $m \subset I$ and $m \rightarrow \infty$ for $h=h_{m}$ and $k=k_{m}$ in the equations (1.5) and (1.6), we find that $U(x, t)$ satisfies (1.1) and (1.2) in $R_{h_{\infty}}^{T}$ and in $R^{T}$. Thus we obtain that, if $u_{10}, u_{20}, u_{30}$ and $u_{40}$ are of class $\mathscr{B}^{4}, \mathscr{B}^{4}, \mathscr{B}^{3}$ and $\mathscr{B}^{3}$ respectively, the limit function $U(x, t)$ is a solution of (1.1) and (1.2) such that

$$
\begin{aligned}
& u_{1}(x, t) \text { and } u_{2}(x, t) \text { are of class } \mathcal{E}_{t}^{0}\left(\mathscr{B}^{2}\right) \cap \mathcal{E}_{t}^{1}\left(\mathscr{B}^{0}\right), \\
& u_{2}(x, t) \text { and } u_{2}(x, t) \text { are of class } \mathcal{E}_{t}^{0}\left(\mathscr{B}^{1}\right) \cap \mathcal{E}_{t}^{2}\left(\mathscr{B}^{0}\right) \\
& \text { in } R^{T} .
\end{aligned}
$$

Further, if we use the properties of the fundamental solution of heat equation, we can modify that, if $u_{10}, u_{20}, u_{30}$ and $u_{40}$ are of class $\mathscr{B}^{2}, \mathscr{B}^{2}, \mathscr{B}^{1}$ and $\mathscr{B}^{1}$ respectively, the same results (2.16) are obtained.

Thus we have the following Theorem;

\section{Theorem 1.}

If $u_{10}, u_{20}, u_{30}$ and $u_{40}$ are of class $\mathscr{B}^{2}, \mathscr{B}^{2}, \mathscr{B}^{1}$ and $\mathscr{B}^{1}$ respectively and they are all non-negative, then there exists the non-negative solution, which satisfies (2.16), of the problem (1.1) and (1.2). 


\section{$\S 3$. Uniqueness Theorem}

We have the following lemma.

\section{Lemma 2 (Convergence).}

If $U(x, t)$ is a solution of (1.1) and (1.2), for which $u_{1}(x, t)$ and $u_{2}(x, t)$ are of class $\mathcal{E}_{t}^{0}\left(\mathscr{B}^{2}\right) \cap \mathcal{E}_{t}^{1}\left(\mathscr{B}^{0}\right)$, and $u_{3}(x, t)$ and $u_{4}(x, t)$ are of class $\mathcal{E}_{t}^{\mathrm{l}}\left(\mathscr{B}^{0}\right)$ in $R^{T}$, and if $U^{n, j}$ is the solution of (2.1) and (2.2) under the assumption of Lemma 1 , then there exists a $\delta(\varepsilon)$ for any $\varepsilon$, such that for $(x=j h, t=n k)$ in $R_{h}^{T}$ and $0<h, k \leqslant \delta$

$$
\left\|U^{n, j}-U(x, t)\right\|<\varepsilon
$$

where

$$
\|u\|=\sup _{R_{h}^{T}}|u(x, t)| \quad \text { and } \quad\|U\|=\sum_{i=1}^{4}\left\|u_{i}\right\| .
$$

Proof. We have

$$
\begin{aligned}
P\left(u_{1}(x, t)\right)= & \lambda u_{1}(x+h, t)+(1-2 \lambda) u_{1}(x, t)+\lambda u_{1}(x-h, t) \\
= & u_{1}(x, t)+\frac{\lambda h^{2}}{2}\left\{\frac{\partial^{2}}{\partial x^{2}} u_{1}(x+\theta h, t)+\frac{\partial^{2}}{\partial x^{2}} u_{1}\left(x-\theta^{\prime} h, t\right)\right\} \\
\quad\left(0<\theta, \theta^{\prime}<1\right) & \\
= & u_{1}(x, t)+k\left\{\frac{\partial}{\partial t} u_{1}(x, t)+d_{1} u_{1}(x, t) u_{4}(x, t)\right. \\
& \left.+d_{2} u_{1}(x, t) u_{3}(x, t)\right\}+0(k) \\
= & u_{1}(x, t+k)+k\left\{d_{1} u_{1}(x, t) u_{4}(x, t)+d_{2} u_{1}(x, t)\right. \\
& \left.u_{3}(x, t)\right\}+0(k) \\
= & u_{1}(x, t+k)+k\left\{d_{1} u_{1}(x, t+k) u_{4}(x, t)\right. \\
& \left.+d_{2} u_{1}(x, t+k) u_{3}(x, t)\right\}+0(k) .
\end{aligned}
$$

Thus, it follows that

$$
\begin{aligned}
u_{1}(x, t+k)= & P\left(u_{1}(x, t)\right)-k\left\{d_{1} u_{1}(x, t+k) u_{4}(x, t)\right. \\
& \left.+d_{2} u_{1}(x, t+k) u_{3}(x, t)\right\}+0(k) .
\end{aligned}
$$

By similar calculation we have

$$
\begin{aligned}
u_{2}(x, t+k)= & P\left(u_{2}(x, t)\right)-k\left\{d_{3} u_{2}(x, t+k) u_{4}(x, t)\right. \\
& \left.-d_{2} u_{1}(x, t+k) u_{3}(x, t)\right\}+0(k), \\
u_{3}(x, t+k)= & u_{3}(x, t)+k\left\{d_{3} u_{2}(x, t) u_{4}(x, t+k)\right. \\
& \left.-d_{2} u_{1}(x, t) u_{3}(x, t+k)\right\}+0(k),
\end{aligned}
$$




$$
\begin{aligned}
u_{4}(x, t+k)= & u_{4}(x, t)-k\left\{d_{1} u_{4}(x, t+k) u_{1}(x, t)\right. \\
& \left.+d_{3} u_{2}(x, t) u_{4}(x, t+k)\right\}+0(k) .
\end{aligned}
$$

Putting $s_{i}(x, t)=u_{i}^{n, j}-u_{i}(x, t)$ for $i=1,2,3,4$, we get the following difference scheme from (3.1), (3.2), (3.3) and (3.4),

$$
\begin{aligned}
& s_{1}(x, t+k)=\frac{P\left(s_{1}(x, t)\right)-k f_{1}+0(k)}{1+k\left(d_{1} u_{4}^{n, j}+d_{2} u_{3}^{n, j}\right)} \\
& s_{2}(x, t+k)=\frac{P\left(s_{2}(x, t)\right)-k f_{2}+0(k)}{1+k d_{3} u_{4}^{n, j}} \\
& s_{3}(x, t+k)=\frac{s_{3}(x, t)-k f_{3}+0(k)}{1+k d_{2} u_{1}^{n, j}} \\
& s_{4}(x, t+k)=\frac{s_{4}(x, t)-k f_{4}+0(k)}{1+k\left(d_{1} u_{1}^{n, j}+d_{3} u_{2}^{n, j}\right)}
\end{aligned}
$$

in $R_{h}^{T}$ with the initial data

$$
S(x, 0)=0
$$

where $S={ }^{t}\left(s_{1}, s_{2}, s_{3}, s_{4}\right)$ and

$$
\begin{aligned}
f_{1}= & \left\{d_{1} s_{4}(x, t)+d_{2} s_{3}(x, t)\right\} u_{1}(x, t+k), \\
f_{2}= & d_{3} u_{2}(x, t+k) s_{4}(x, t)-d_{2} u_{1}(x, t+k) s_{3}(x, t) \\
& -d_{2} u_{3}^{n, j} s_{1}(x, t+k), \\
f_{3}= & -d_{3} u_{4}(x, t+k) s_{2}(x, t)+d_{2} u_{3}(x, t+k) s_{1}(x, t) \\
& -d_{3} u_{2}^{n, j} s_{4}(x, t+k), \\
f_{4}= & \left\{d_{1} s_{1}(x, t)+d_{3} s_{2}(x, t)\right\} u_{4}(x, t+k) .
\end{aligned}
$$

Here we consider the following difference scheme for $Y={ }^{t}\left(y_{1}, y_{2}\right.$, $\left.y_{3}, y_{4}\right)$

$$
\begin{aligned}
& y_{1}(x, t+k)=P\left(y_{1}(x, t)\right)+k\left(F_{1}+G_{1}\right) \\
& y_{2}(x, t+k)=P\left(y_{2}(x, t)\right)+k\left(F_{2}+G_{2}\right) \\
& y_{3}(x, t+k)=\quad y_{3}(x, t)+k\left(F_{3}+G_{3}\right) \\
& y_{4}(x, t+k)=\quad y_{4}(x, t)+k\left(F_{4}+G_{4}\right)
\end{aligned}
$$

in $R_{h}^{T}$ with the initial data

$$
Y(x, 0)=0,
$$

where $F_{i}=\sup _{R_{h}^{T}}\left|f_{i}(x, t, k)\right| . \quad G_{i}$ is the upper bound of $g_{i}$ and $g_{i}$ $=g_{i}(x, t, k)$ tends to 0 as $k \rightarrow 0$ for $i=1,2,3,4$ 。 
We have the following equations from (3.8) and (3.9),

$$
y_{i}(x, t)=t\left(F_{i}+G_{i}\right) \quad \text { for } \quad i=1,2,3,4 \text {. }
$$

Therefore we get the following inequalities from (3.5) and (3.9)

$$
\left|s_{i}(x, t)\right| \leqslant y_{i}(x, t) \quad \text { for } \quad i=1,2,3,4
$$

and then we have in $0 \leqslant t \leqslant T$

$$
\left\|s_{i}\right\| \leqslant T\left(F_{i}+G_{i}\right) \quad \text { for } \quad i=1,2,3,4 .
$$

Thus, from (3.12) we have

$$
\|S\| \leqslant \sum_{i=1}^{4} T\left(F_{i}+G_{i}\right) .
$$

On the other hand, we find from (3.7) that

$$
\sum_{i=1}^{4} F_{i} \leqslant 3 D M\|S\| \text {. }
$$

Then we get

$$
(1-3 D M T)\|S\| \leqslant T \sum_{i=1}^{4} G_{i} \text {. }
$$

If we select $T=T_{1}$ such that $T_{1}<1 / 3 D M$, there exists a $\delta(\varepsilon)$ for any $\varepsilon$ such that $0<h, k \leqslant \delta$ and

$$
\|S\|<\varepsilon \quad \text { in } \quad 0 \leqslant t \leqslant T_{1} .
$$

If we consider $t=T_{1}$ as a new initial time, we get $t=T_{2}$ such that $T_{2}<T_{1}+1 / 3 D M$ and

$$
\|S\|<\varepsilon \quad \text { in } \quad T_{1} \leqslant t \leqslant T_{2} .
$$

Thus, if we continue the same argument, we have the same result as (3.16) in $R_{h}^{T}$ and Lemma 2 is proved.

Here if $U_{1}(x, t)$ and $U_{2}(x, t)$ are both arbitrary functions satisfying the assumption of Lemma 2, then there exists a $\delta(\varepsilon)$ for any $\varepsilon$ such that $0<h, k \leqslant \delta$ implies

$$
\begin{aligned}
\left\|U_{1}(x, t)-U_{2}(x, t)\right\| & \leqslant\left\|U_{1}(x, t)-U^{n, j}\right\|+\left\|U_{2}(x, t)-U^{n, j}\right\| \\
& \leqslant 2 \varepsilon \text { in } R_{h}^{T},
\end{aligned}
$$

where $U^{n, j}$ is the solution of (1.5) and (1.6).

Thus, we have proved the following theorem; 


\section{Theorem 2.}

As for a genuine solution $U(x, t)$ of (1.1) and (1.2) satisfying the assumption of Lemma 2 , the solution is unique.

Remark. Similarly we can deal with the following system of an immobilizing reaction of higher order,

$$
\begin{aligned}
& \frac{\partial U}{\partial t}=D \frac{\partial^{2} U}{\partial x^{2}}+A\left(U^{P}\right) U \\
& U(x, 0)=U_{0}(x)
\end{aligned}
$$

where for non-negative integer $P$,

$$
A\left(U^{P}\right)=\left(\begin{array}{cccc}
-d_{2} u_{1}^{p_{1}} u_{3}^{p_{3}} & 0 & 0 & -d_{1} u_{1}^{p_{5}} u_{4}^{p_{6}} \\
d_{2} u_{1}^{p_{1}} u_{3}^{p_{3}} & -d_{3} u_{2}^{p_{2}} u_{4}^{p_{4}} & 0 & 0 \\
-d_{2} u_{1}^{p_{1}} u_{3}^{p_{3}} & d_{3} u_{2}^{p_{2}} u_{4}^{p_{4}} & 0 & 0 \\
0 & -d_{3} u_{2}^{p_{2}} u_{4}^{p_{4}} & 0 & -d_{1} u_{1}^{p_{5}} u_{4}^{p_{6}}
\end{array}\right)
$$

and prove the existence and the uniqueness of the solution.

\section{Acknowledgement}

The author wishes to thank Professor M. Yamaguti who gave him valuable suggestions and many criticisms.

\section{References}

[1] Arima. R. and Y. Hasegawa, On global solutions for mixed problem of a semilinear differential equation, Proc. Japan Acad. 139 (1963), 721-725.

[2] Brian, P. L. T., etc., Penetration theory for a gas absorption accompanied by a second order chemical reaction, A. I. Ch. E. Journal 7226 (1961), 226-231.

[3] Courant, R., K. Friedrichs and H. Lewy; Uber die Partiellen Differenzengleichungen der mathematischen Physik, Math. Ann. 100 (1928), 215-234.

[4] Crank, J., Mathematics of Diffusion, Clarendon Press, Oxford, 1955.

[5] Forsythe, G. E. and W. R. Wasow, Finite Difference Methods for Partial Differential Equation, Wiley, 1960.

[6] John, F., On integration of parabolic equations by difference methods, Comm. Pure Appl. Math. 5 (1952), 155-210.

[7] Nogi, T., Private communication (1964). 